\title{
Akt Pathway Inhibition of the Solenopsin Analog, 2-Dodecylsulfanyl-1,-4,-5,-6-tetrahydropyrimidine
}

\author{
NNE E. UKO ${ }^{1}$, OSMAN F. GÜNER ${ }^{2}$, J. PHILLIP BOWEN ${ }^{1}$ and DIANE F. MATESIC ${ }^{1}$ \\ ${ }^{1}$ Department of Pharmaceutical Sciences, College of Pharmacy, Mercer University, Atlanta, GA, U.S.A.; \\ ${ }^{2}$ Department of Chemistry and Physics, Santa Rosa Junior College, Santa Rosa, CA, U.S.A.
}

\begin{abstract}
Background/Aim: The P13K/Akt signaling pathway is a growth-regulating cellular pathway that is constitutively activated in a variety of human cancers. In previous studies, we reported that a solenopsin analog, compound $\boldsymbol{B}(M U-06-$ SC-608-7), shows inhibitory effects on Akt phosphorylation at a key activation site, as well as on proliferation of tumorigenic cells at sub-micromolar concentrations. The purpose of this study was to evaluate the effect of compound $\boldsymbol{B}$ on downstream effectors of Akt kinase, phosphorylation of Akt at a second activation site, Akt kinase activity in vitro, tumorigenic cell viability and other signaling pathways. Materials and Methods: Western blot analyses were performed using WBras 1 epithelial and H2009 human carcinoma cells and cell viability assays were performed on $\mathrm{H} 2009$ cells. In vitro Akt kinase assays were performed using a commercially available kit. Results: Compound $\boldsymbol{B}$ decreased the phosphorylation of Akt at the Thr308 activation site and key downstream effectors of Akt kinase, but did not directly inhibit Akt kinase. Substantial decreases in cell viability were observed at concentrations above $5 \mu M$. No effect was seen on ERK or JNK pathways. Conclusion: The results earmark this compound for further studies as a potential targeted cancer therapy.
\end{abstract}

Akt kinase is recognized as the critical signal regulatory hub for cell growth, survival, metabolism, and proliferation (1-3). The pathogenesis of numerous human cancers is plagued with aberrant regulation of the Akt pathway, which is downstream of phosphatidylinositol-4,5-bisphosphate-3-kinase (PI3K) (4$6)$. Inhibitors for multiple targets along this pathway are in clinical trials for the treatment of cancer (6-8).

This article is freely accessible online.

Correspondence to: Diane F. Matesic, Department of Pharmaceutical Sciences, Mercer University, 3001 Mercer University Drive, Atlanta, GA 30341, U.S.A. Tel: +1 6785476241, Fax: +1 6785476423, email: matesic_df@mercer.edu

Key Words: Akt, solenopsin A, MDM2, perifosine, ERK.
As a signal transduction protein, Akt kinase is responsible for the phosphorylation of several downstream substrates, which propagate signals through the cytoplasm to carry out cellular functions. These include: i) glycogen synthetase kinase-3beta (GSK3 3 ), ii) murine double minute 2 (MDM2), iii) apoptosis signal-regulating kinase 1 (ASK1), iv) Bcl-X $\mathrm{L} / \mathrm{Bcl}$-2-associated death promoter (BAD), v) forkhead protein (FOXO), and vi) tuberin/TSC2 (3).

Akt kinase binds to PIP3 via its PH domain during its recruitment to the plasma membrane, where it requires phosphorylation at two active sites for its full activation (9, 10). The interaction of Akt with the phospholipid at the membrane induces a conformational change, and exposes the Thr308 Akt residue for activation $(11,12)$. Thr308 is located on the activation loop of the bound Akt kinase and is phosphorylated by PDK1, which partially activates the Akt kinase (13-15). Following this partial activation, mammalian target of rapamycin complex 2 (mTORC2) facilitates the full activation of Akt by phosphorylation of the second Akt activation site, Ser473, at the C-terminal regulatory domain in the hydrophobic motif region $(9,16)$.

Hyperactivity of Akt is implicated in several human cancers, such as breast, lung, ovarian, prostate, renal, glioma, and pancreatic, resulting in aberrant cell replication, growth, metabolism and other cellular functions $(5,6)$. The hyperactivity of Akt leads to mTORC1 stimulation with a subsequent increase in cell division overriding the checkpoint for halting aberrant cell division, resulting in the accumulation of mutations and the eventual development of malignancies. The amplification and overexpression of Akt suppresses FOXO by phosphorylation, causing stimulation of cell proliferation. Likewise, GSK3 $\beta$ is inactivated through phosphorylation by Akt, thereby allowing $\mathrm{G}_{1}$ cell-cycle progression without restriction and increased translation (6).

Akt has been recognized as a target for anticancer therapy and various small molecular inhibitors are in clinical development for this purpose. ATP competitive inhibitors, namely GDC0068 (ipatasertib), GSK795 (uprosertib), AZD5363, A-443654 are in clinical assessment phase II; also, at this stage are the allosteric Akt 


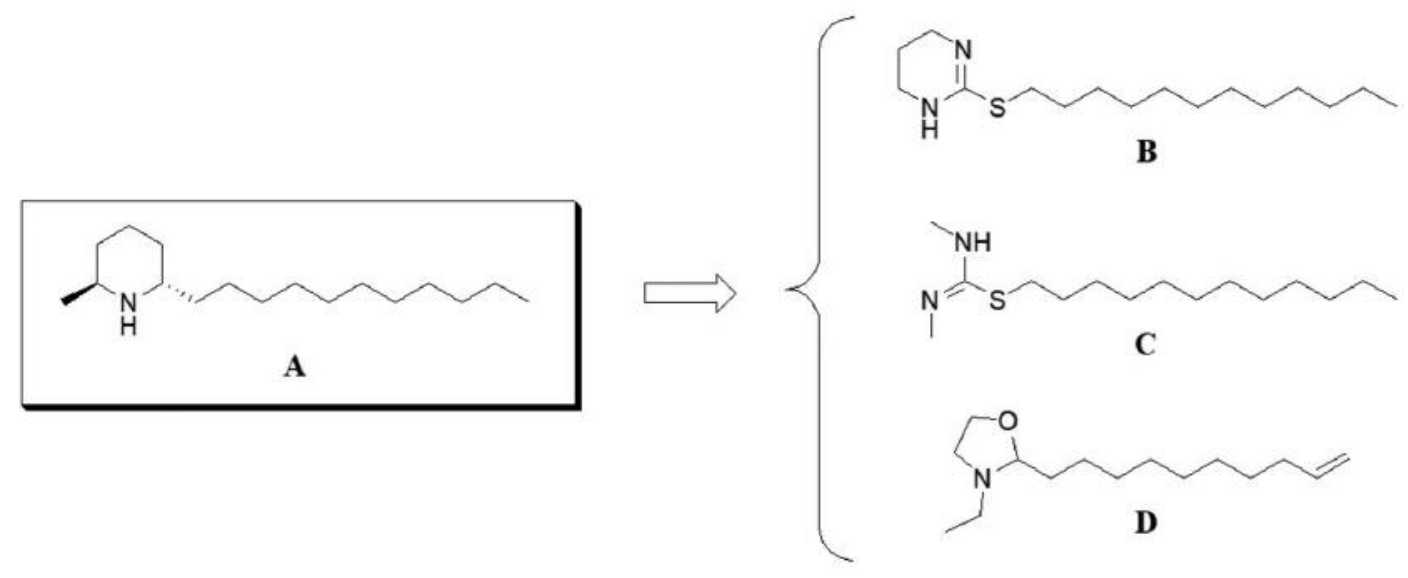

Figure 1. Through pharmacophore modeling based on $\boldsymbol{A}$ (solenopsin A), three commercially available compounds $(\boldsymbol{B}, \boldsymbol{C}, \boldsymbol{D})$ were identified as potential Akt pathway inhibitors. Compound $\boldsymbol{B}$ has been shown to inhibit Akt activation.

inhibitors, such as perifosine (KRX-0401), MK2206, and ARQ $092(7,8,17)$.

Solenopsin A, an alkaloid component of fire ant venom, has been reported to be an Akt inhibitor that can antagonize Akt cellular activity in vitro $(18,19)$. Since it is a competitive inhibitor and seems to exert inhibitory effects at fairly high concentrations (19), we have previously modeled and selected novel analogs of solenopsin A, shown in Figure 1 , using pharmacophore modeling in an attempt to enhance the efficacy (20). In those studies, we reported a lead solenopsin analog, compound $\mathbf{B}$, with inhibitory effects on Akt phosphorylation at a major activation site as well as on the proliferation of tumorigenic cells (20). Now, we demonstrate Akt pathway downstream effects, effects on the Akt Thr308 activation site, effects on cell viability and an in vitro Akt kinase assay of compound $\mathbf{B}$.

\section{Materials and Methods}

Materials. $\mathrm{H} 2009$ human lung tumor cells from the American Type Culture Collection (ATCC, Manassas, VA, USA) were provided by Randall Ruch at the University of Toledo. WB-ras rat liver epithelial cells obtained from J. Trosko (Michigan State University) were subcloned from single cells to obtain the WBras 1 line. Pharmacophore modeled compound $\mathbf{B}$ was obtained commercially from Enamine Ltd. RPMI Medium 1640(1X), L-glutamine, trypsin and phosphate buffered saline (PBS) were purchased from Fisher Scientific (Pittsburgh, PA, USA), and fetal bovine serum (FBS) was obtained from Invitrogen (Carlsbad, CA, USA). Tissue culture treated flasks with vented caps were obtained from CELLTREAT Scientific Products (Pepperrell, MA, USA). Phenylmethylsulfonyl fluoride (PMSF), Trypan blue solution, protease inhibitor cocktail, G418, DMSO, BSA and Ponceau Red solution were from Sigma Aldrich (St. Louis, MO, USA), Pan-Akt, phospho-Akt (Ser473), phospho-Akt (Thr308), PTEN, PDK1, phosphoPDK1 (ser241), mTOR, phospho-mTOR (Ser2481), and phospho-JNK (Thr183/Tyr185), phospho-PTEN (ser308/thr382,383), phospho-
GSK3 $\beta$ (Ser9), phospho-MDM2 (ser166), phospho-P70S6 Kinase (Thr389), phospho-4E-BP1 (Thr37/46), $\beta$-actin, $\alpha$-tubulin, anti-rabbit IgG alkaline phosphatase-conjugated and, anti-rabbit IgG horseradish peroxidase-conjugated antibodies were purchased from Cell Signaling Technology (Beverly, MA, USA). Nonfat dry milk, Tris-hydrochloride, Tween-20, DC Protein Assay, Sodium Dodecyl Sulfate (SDS), 25x alkaline phosphatase color development buffer, 5-bromo-4-chloro-3indolyl phosphate/nitroblue tetrazolium (BCIP/NBT), protein molecular mass standards, PVDF membrane packs and all electrophoresis and transfer buffer components were purchased from Bio-Rad (Hercules, CA, USA). All other solvents, reagents and chemicals used were of analytical grade.

Cell culture. WBras 1 cells were grown in alpha Modification of Eagle's Medium supplemented with $2 \mathrm{mM}$ L-glutamine and 5\% FBS, and used for experiments between passages 7-12. G418 antibiotic was added to the $\alpha$-MEM for culturing cells at a concentration of $500 \mu \mathrm{M}$, but was not added to cells seeded for experiments. Human lung carcinoma cells (H2009) were grown in RPMI-1640 liquid media with $2 \mathrm{mM} \mathrm{L-glutamine} \mathrm{and} \mathrm{5 \%} \mathrm{fetal}$ bovine serum added, and were used between passages 38-51. Cells were subcultured by trypsinization and were seeded at $5-10 \%$ confluency in $25 \mathrm{~cm}^{2}$ flasks and were incubated at $37^{\circ} \mathrm{C}$ in an atmosphere of $5 \% \mathrm{CO}_{2}$. At $75-90 \%$ confluency, cells were treated with vehicle dimethylsulfoxide (DMSO) or test compounds and reincubated for varying times as indicated.

Extraction of proteins. Treated cells were washed with $10 \mathrm{ml}$ of PBS and were lysed with $250 \mu \mathrm{l} 2 \%$ SDS containing $1 \mathrm{mM}$ PMSF, and 1:100 dilution of protease inhibitor cocktail. The lysates were scraped and transferred into microcentrifuge tubes and were sonicated for two 15 second pulses at room temperature. Unused samples were frozen in liquid nitrogen and stored at $-20^{\circ} \mathrm{C}$.

Protein concentration assay. Bio-Rad (Hercules, CA, USA) DC protein assay was utilized to determine the protein concentrations of the sonicated cell extracts. Bovine serum albumin (BSA) was used as a standard and the absorbance of dye was read at $750 \mathrm{~nm}$ using a TECAN (Mannedorf, Switzerland) GENios plate reader. 
A

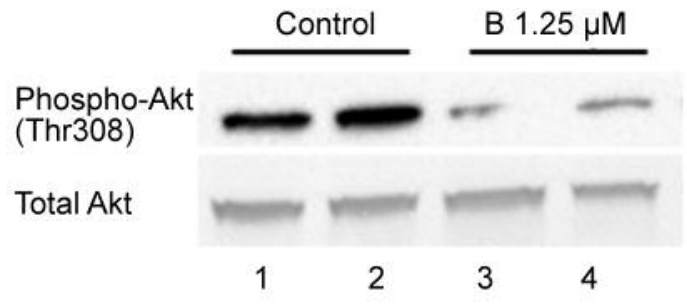

C

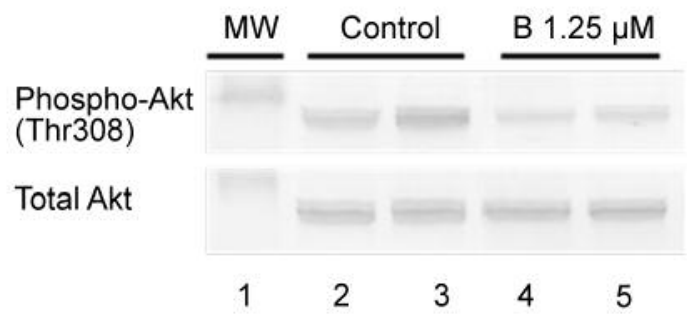

E

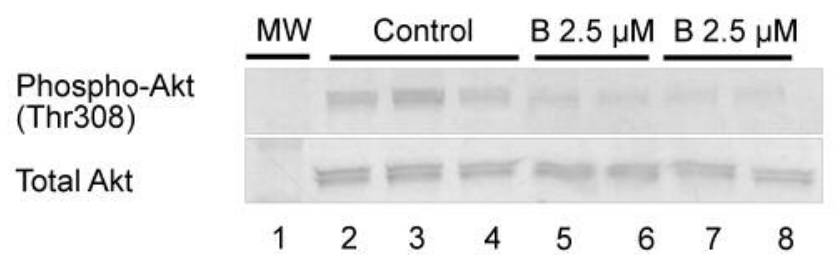

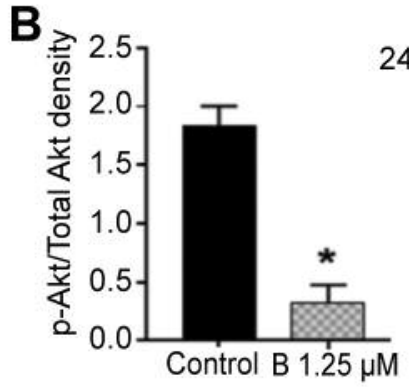

$24 \mathrm{~h}$

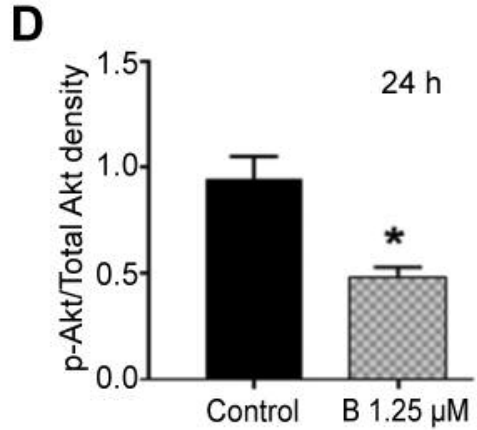

$\mathbf{F}$

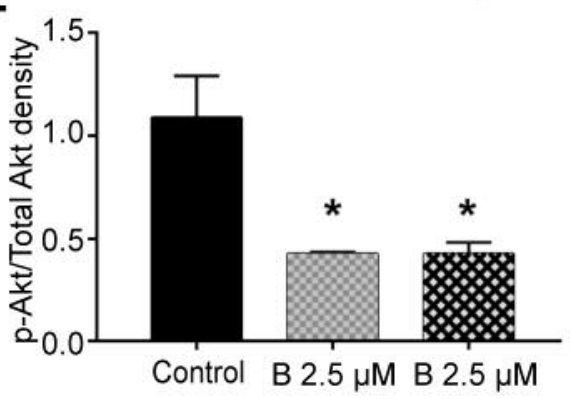

Control

B $1 \mathrm{~h}$

$\infty$ B $4 h$

Figure 2. Effect of compound $\boldsymbol{B}$ on Akt kinase phosphorylation in WBras1 cells (A and B) or in H2009 cells (C and D) at 24 h following treatment. Immunoblotting of whole cell lysates shows the effect of compound $\boldsymbol{B}$ on Akt (Thr308) phosphorylation or total Akt (bottom lanes). Treatment groups were: Lane 1, pre-stained molecular weight marker, vehicle (lanes 1-2), $1.25 \mu M$ compound $\boldsymbol{B}$ (lanes 3-4). (E and F) Effect of compound B on Akt kinase phosphorylation at Thr308 in $H 2009$ cells at 1 or 4 h following treatment. Treatment groups were: Lane 1, pre-stained molecular weight marker, vehicle (lanes 2-4), $2.5 \mu \mathrm{M}$ compound $\boldsymbol{B}$ at 1 h (lanes 5-6), $2.5 \mu \mathrm{M}$ compound $\boldsymbol{B}$ at 4 h (lanes 7-8). All samples in E and D received matched amounts of vehicle for $4 \mathrm{~h}$. Densitometric quantification of bands shown in the graphs to the right represents the mean $\pm S . D$. ( $p<0.05$ compared to control).

Western immunoblot assay. For electrophoresis of protein samples, $4 \mathrm{x}$ Laemmli sample buffer was added to equal amounts of protein per lane at $25 \%$ of the final volumes, separated on $12.0 \%$ acrylamide SDS gels, and transferred semi-dry to PVDF membranes utilizing Bio-Rad Trans-Blot Turbo for 7-10 min. Membranes were washed for 1 min using deionized $\mathrm{H}_{2} \mathrm{O}$, stained with Ponceau Red for 3-10 minutes, washed with deionized $\mathrm{H}_{2} \mathrm{O}$ five times, scanned, and blocked using $4 \%$ non-fat dry milk, $0.1 \%$ Tween-20, $40 \mathrm{mM}$ Tris, $\mathrm{pH} 7.5$ for 60 minutes. Specific primary antibodies were incubated with blots in blocking buffer overnight at $4^{\circ} \mathrm{C}$ on a shaker. Using horseradish peroxidase or alkaline phosphatase anti-rabbit secondary antibody, immunopositive bands were detected and developed using chemiluminescence or the color development substrate, BCIP/NBT. Reprobes of selected blots were performed by 1-2 second rehydration in methanol, followed by $30-\mathrm{min}$ incubation in blocking buffer, primary antibody incubation, secondary antibody incubation and development, as described above. The blots were air dried, then scanned on a HP Scanjet 

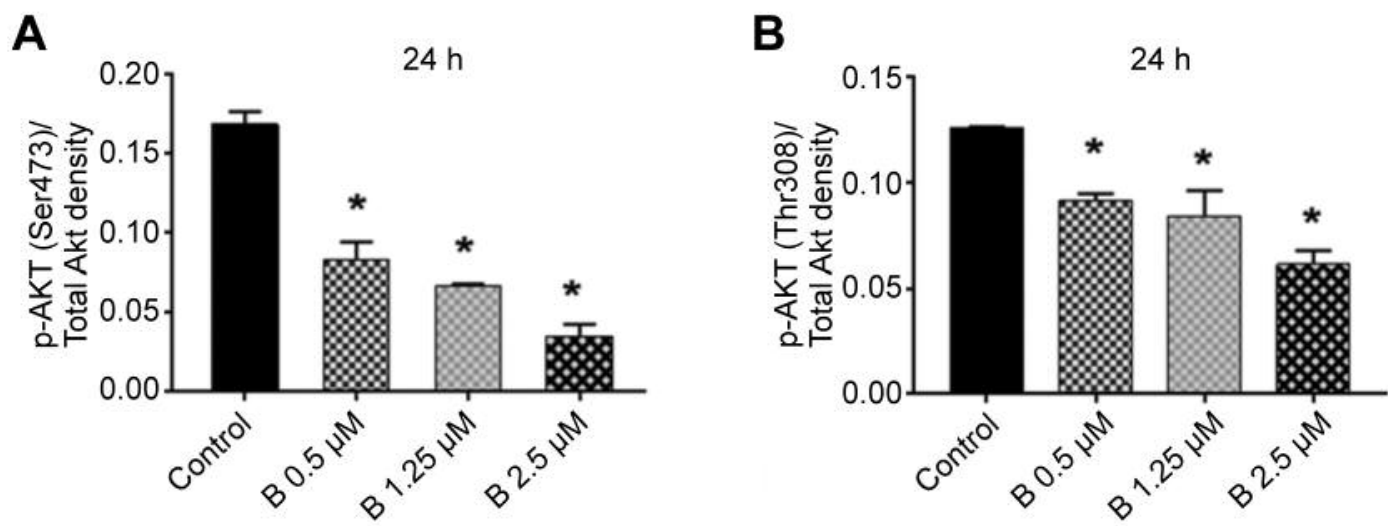

Figure 3. Dose-dependent effect of compound $\boldsymbol{B}$ on Akt kinase in $H 2009$ cells at 24 h treatment. Immunoblotting of whole lysates analysis indicated the effect of compound $\boldsymbol{B}$ on (A) Akt Ser473 or (B) Thr308 phosphorylation normalized total Akt. Densitometric quantification of bands shown in the graphs represents the mean $\pm S . D .(p<0.05$ compared to controls).

4400C (Hewlett-Packard, Palo Alto, CA, USA) scanner. To measure band intensity, UN-SCAN-IT software (version 6.1) from Silk Scientific, Inc. (Orem, UT, USA) was utilized and statistical analyses were performed with Statistix ${ }^{\circledR}$ (Analytical Software, Tallahassee, FL, USA) software.

Cell viability assay. $\mathrm{H} 2009$ cells were sub-cultured into 96-well plates, incubated for $48 \mathrm{~h}$ at $37^{\circ} \mathrm{C}$ and $5 \% \mathrm{CO}_{2}$ to allow cell attachment and were grown to approximately $75 \%$ confluence before treatment. Medium was changed after $24 \mathrm{~h}$ before treating the cells with vehicle or compound $\mathbf{B}$ and were incubated for an additional $22 \mathrm{~h}$. Next, addition of the 3-(4, 5-dimethylthiazol-2-yl)2, 5-diphenyltetrazolium bromide (MTT, Cell Applications, Inc., San Diego, CA) solution to each well was followed by incubation for $4 \mathrm{~h}$ in a cell culture incubator. The supernatant was removed, and the formazan crystals were dissolved using SDS and were incubated at room temperature for 5-15 minutes before reading the absorbance at $360 \mathrm{~nm}$ using a microplate spectrophotometer.

Akt kinase activity assay. The Cyclex AKT/PKB kinase/Inhibitor Screening Kit (CY-1168) (Woburn, MA, USA) was used to measure and evaluate the activity of Akt in a cell-free assay. The 96-well plate provided by the manufacturer is pre-coated with a peptide substrate of Akt kinase, AKTide-2T, which is actively phosphorylated by isoforms of Akt. $2.5 \mu \mathrm{L}$ of vehicle (DMSO) or compound $\mathbf{B}$ was added into separate quadruplet wells, followed by the addition of $100 \mu \mathrm{L} /$ well of reaction buffer and an incubation at $30^{\circ} \mathrm{C}$ for $1 \mathrm{~h}$. Following the wash step in $1 \mathrm{X}$ wash buffer, $100 \mu \mathrm{l}$ of HRP conjugated anti-phosphoAKTide-2T monoclonal antibody was added to the wells and was incubated at room temperature for $1 \mathrm{~h}$. After washing in $1 \mathrm{X}$ wash buffer, $100 \mu \mathrm{l}$ of the substrate was added to react with the HRP conjugate. Subsequently, the stop solution was added to the wells before reading the absorbance at $450 \mathrm{~nm}$. The positive control wells received $90 \mu \mathrm{l}$ kinase reaction buffer (containing ATP), $5 \mu \mathrm{l} \mathrm{Akt}$ enzyme and $2.5 \mu \mathrm{l}$ vehicle. The test sample wells received $2.5 \mu \mathrm{l}$ compound B, $90 \mu \mathrm{l}$ kinase reaction buffer, and $5 \mu \mathrm{l}$ Akt enzyme. The effect of compound $\mathbf{B}$ was compared to a known Akt inhibitor, staurosporine, provided in the kit. This study was performed in accordance with the well-established Akt Assay kit procedures.
Statistical analyses of data were performed using Statistix for Windows 8 .

\section{Results}

Compound $\boldsymbol{B}$ regulates Akt activation in WBrasl and human carcinoma cells. We have previously reported decreased Akt phosphorylation at the Ser473 activation site by micromolar concentrations of compound $\mathbf{B}$ in both WB-ras 1 and H2009 at 4 and $24 \mathrm{~h}$ following treatment (14). Here, we show regulatory effects of this compound at the Thr308 phosphorylation site of Akt kinase. Figure 2A demonstrates that $1.25 \mu \mathrm{M}$ compound B significantly decreased Thr308 phosphorylation of Akt in WBras 1 cells (See top panel, lanes 3-4, compared to lanes 1-2). Treatment did not substantially change total Akt kinase levels (bottom panel). Quantification of bands (Figure 2B) shows an approximate $75 \%$ decrease in band intensity (phospho-Thr308/total Thr308). In H2009 cells, Figures 2C and D show that 1.25 $\mu \mathrm{M}$ compound $\mathbf{B}$ down-regulated phosphorylation at Akt Thr308 site by about $50 \%$ (top panel, lanes 4-5, compared to lanes 2-3 and graph to the right) at $24 \mathrm{~h}$. Compound B also decreased phosphorylation of the Thr308 site at 1 and $4 \mathrm{~h}$ in $\mathrm{H} 2009$ cells by approximately $65 \%$ (Figures $2 \mathrm{E}$ and F). In these experiments, a higher concentration of compound B $(2.5 \mu \mathrm{M})$ was used to promote a robust response at early times post-treatment. Figure 3 shows the concentration dependence of compound $\mathbf{B}$ on phosphorylation of Akt Ser473 or Thr308 in H2009 cells at $24 \mathrm{~h}$ following treatment. Based on these results, the half maximal inhibitory concentration $\left(\mathrm{IC}_{50}\right)$ is estimated to be $0.6 \mu \mathrm{M}$ for Ser473 and $2.5 \mu \mathrm{M}$ for $\mathrm{Thr} 308$.

Effect of compound $\boldsymbol{B}$ on downstream effectors of Akt in WBrasl and H2009 cells. Figure 4 shows that treatment of 


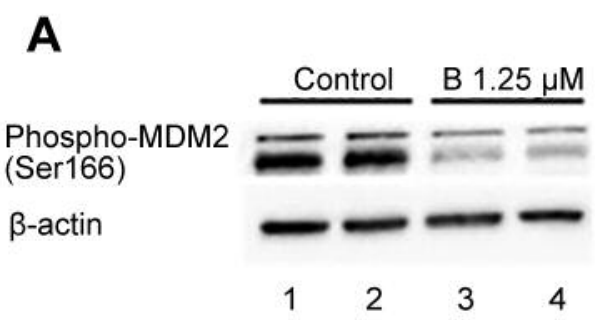

\section{C Phospho-4E-BP1 (Thr37/46)}

$\beta$-actin

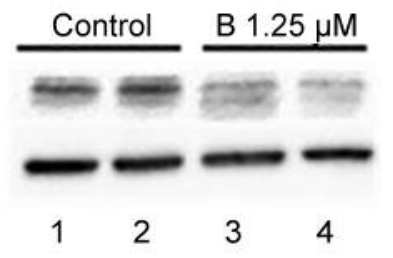

\section{E \\ Phospho-GSK3 $\beta$ (Ser9) \\ $\beta$-actin}
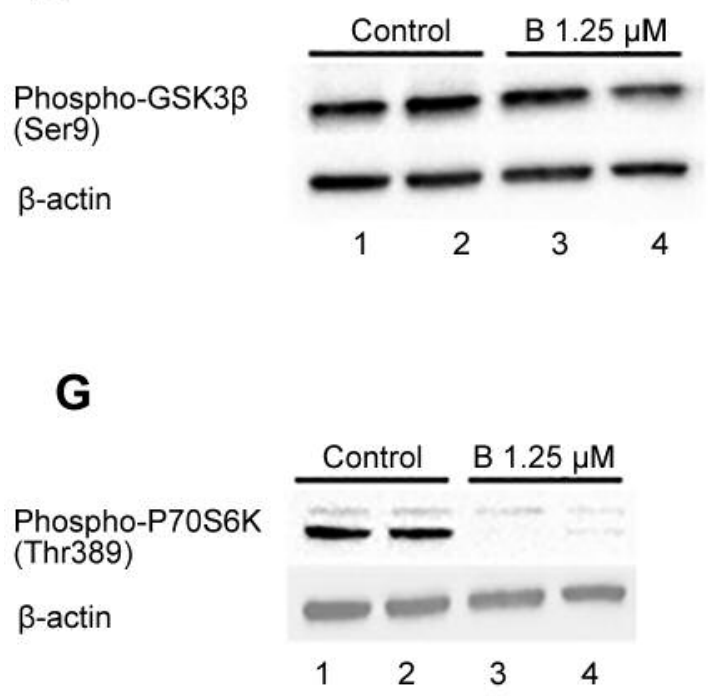
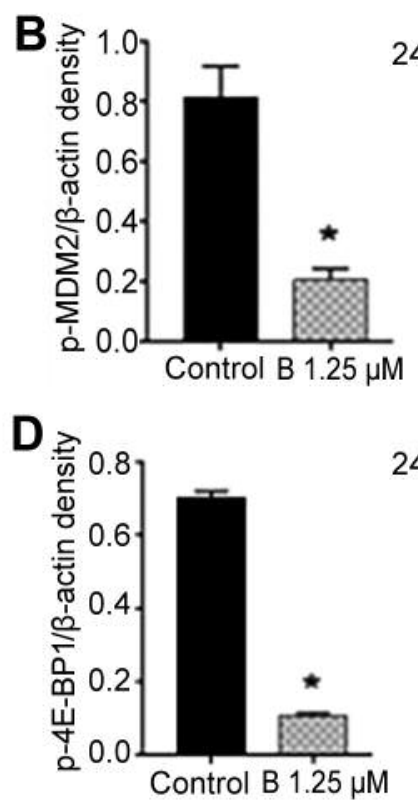

\section{F}
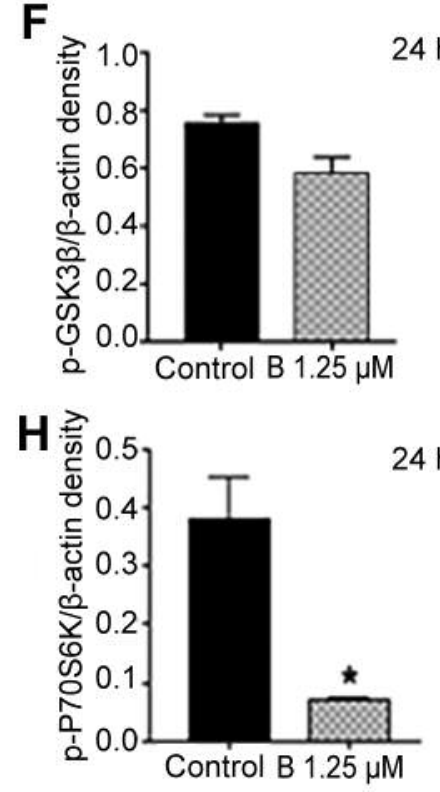

Figure 4. (A and B) Effect of compound $\boldsymbol{B}$ on MDM2 in WBras1 cells at 24 h (h) following treatment. Immunoblotting of whole-lysates shows the effect of compound $\boldsymbol{B}$ on MDM2 (Ser166) phosphorylation or $\beta$-actin (bottom lanes). Treatment groups were: vehicle (lanes 1-2), $1.25 \mu M$ compound $\boldsymbol{B}$ (lanes 3-4). (C and D) Effect of compound $\boldsymbol{B}$ on $4 E-B P 1$ phosphorylation (Thr37/46) in WBras1 cells at 24 h following treatment. (E and F) Effect of compound B on GSK3 $\beta$ (Ser9) phosphorylation in WBrasl cells at 24 h following treatment. Bottom lanes show $\beta$-actin. (G and $H)$ Effect of compound $\boldsymbol{B}$ on P70S6K (Thr389) phosphorylation in WBras1 cells at 24 h following treatment. Bottom lanes show $\beta$-actin. Densitometric quantifications of bands shown in the graphs to the right represent the mean $\pm S . D .(p<0.05$ compared to control).

WBras1 cells for $24 \mathrm{~h}$ with $1.25 \mu \mathrm{M}$ compound $\mathbf{B}$ decreased phosphorylation on the Ser166 activation site of the Akt downstream effector MDM2 by approximately $65 \%$. For 4EBP1 at $24 \mathrm{~h}$, compound $\mathbf{B}$ decreased phosphorylation at the Thr37/46 site by approximately $85 \%$ (Figures $4 \mathrm{C}$ and D).
There was no significant effect on GSK3 $\beta$ (Ser9) phosphorylation at $24 \mathrm{~h}$ (Figures $4 \mathrm{E}$ and F), but reduced phosphorylation of the Akt downstream effector, P70S6 kinase, at the Thr389 site by approximately $80 \%$ was observed (Figures 4G and H). Overall, these results 


\section{A}

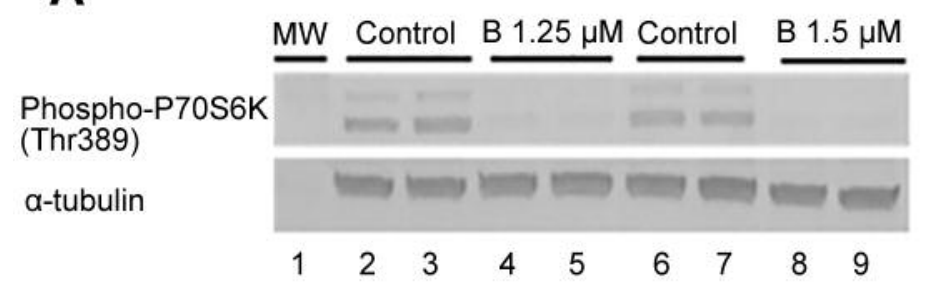

C

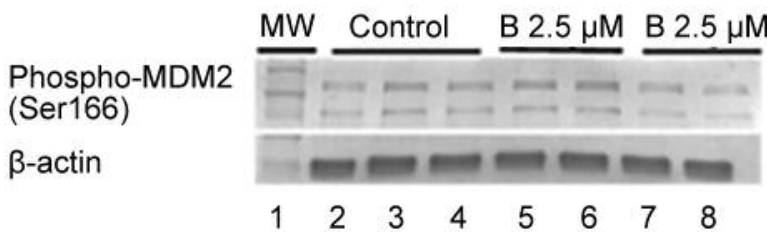

\section{$\mathbf{E}$}

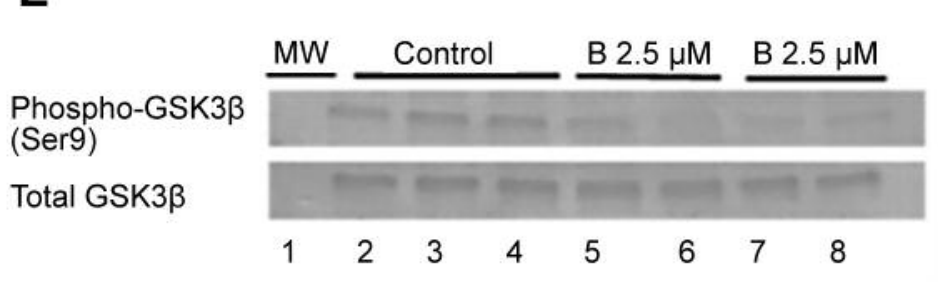

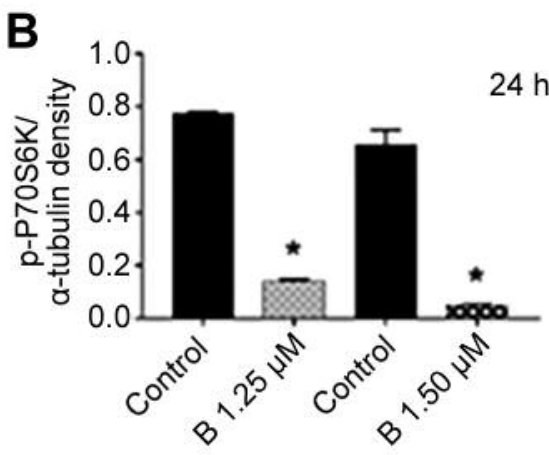

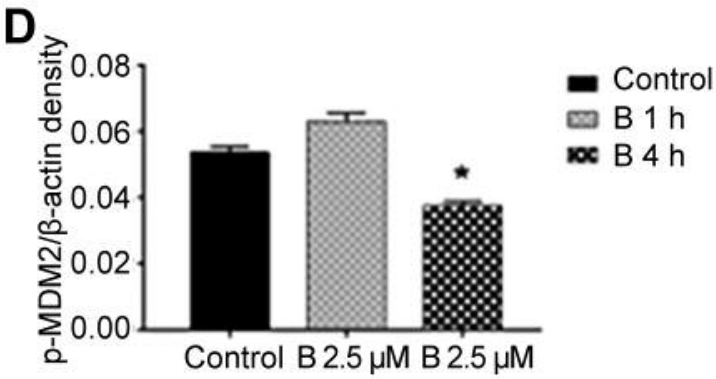

$\mathbf{F}$

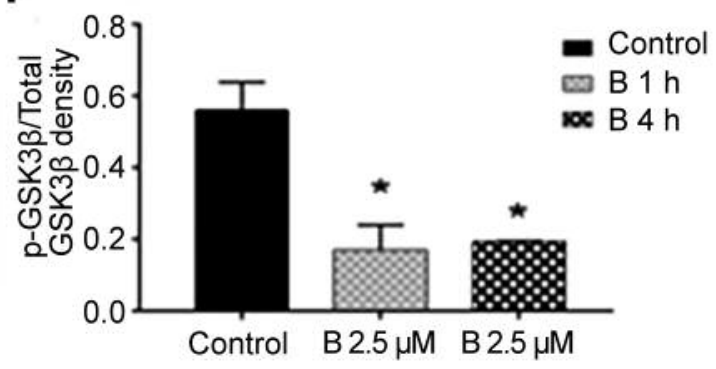

H

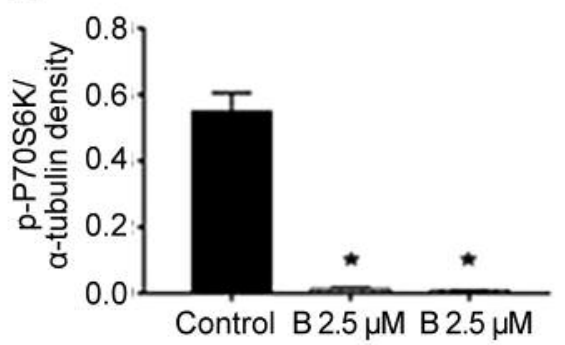

Control 田 $1 \mathrm{~h}$ $14 \mathrm{~h}$ (Thr389) a-tubulin

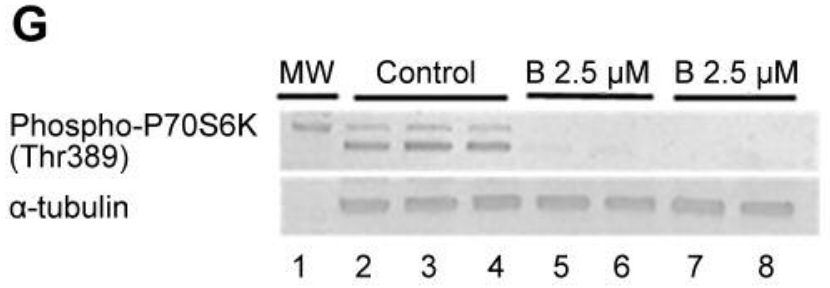

Figure 5. Effect of compound $\boldsymbol{B}$ on downstream effectors of Akt in H2009 cells at 24 h. (A) Western blots of whole-cell lysates show the effect at 24 $h(h)$ on P70S6K (Thr389) phosphorylation (top lanes) or $\alpha$-tubulin (bottom lanes). Treatment groups were: pre-stained molecular weight markers (lane1), vehicle (lanes 2-3), $1.25 \mu$ M compound $\boldsymbol{B}$ (lanes 4-5), vehicle (lane 6-7), $1.5 \mu M$ compound $\boldsymbol{B}$ (lanes 8-9). H). (C and D) Time course effect of compound $\boldsymbol{B}$ on MDM2 (Ser 166) phosphorylation in $\mathrm{H} 2009$ cells at 1 or $4 \mathrm{~h}$ treatment, or $\beta$-actin (bottom lanes). Treatment groups were: vehicle (lanes 2-4), $2.5 \mu M$ compound $\boldsymbol{B}$ at 1 h (lanes 5-6), $2.5 \mu M$ compound $\boldsymbol{B}$ at 4 h (lanes 7-8). (E and F) Time course effect of compound $\boldsymbol{B}$ on GSK3 $\beta$ (ser9) phosphorylation in $H 2009$ cells at 1 or 4 h following treatment, or total GSK3 $\beta$ (bottom lanes). Treatment groups were: pre-stained molecular weight markers (lane1), vehicle (lanes 2-4), $2.5 \mu M$ compound $\boldsymbol{B}$ at $1 \mathrm{~h}$ (lanes 5-6), $2.5 \mu M$ compound $\boldsymbol{B}$ at $4 h$ (lanes 7-8). $(G$ and $H)$ Time course effect of compound $\boldsymbol{B}$ on P70S6K (Thr389) phosphorylation in H2009 cells at 1 or 4 h following treatment, or $\alpha$-tubulin (bottom lanes). Treatment groups were: pre-stained molecular weight marker (lane1), vehicle (lanes 2-4), $2.5 \mu M$ compound $\boldsymbol{B}$ at 1 h (lanes 5-6), $2.5 \mu M$ compound $\boldsymbol{B}$ at $4 \mathrm{~h}$ (lanes 7-8). All samples in $C$ through $H$ received matched amounts of vehicle for $4 h$. (H). Densitometric quantifications of bands shown in the graphs to the right $(B, D, F, H)$ represent the mean $\pm S . D .\left({ }^{*} p<0.05\right.$ compared to control). 

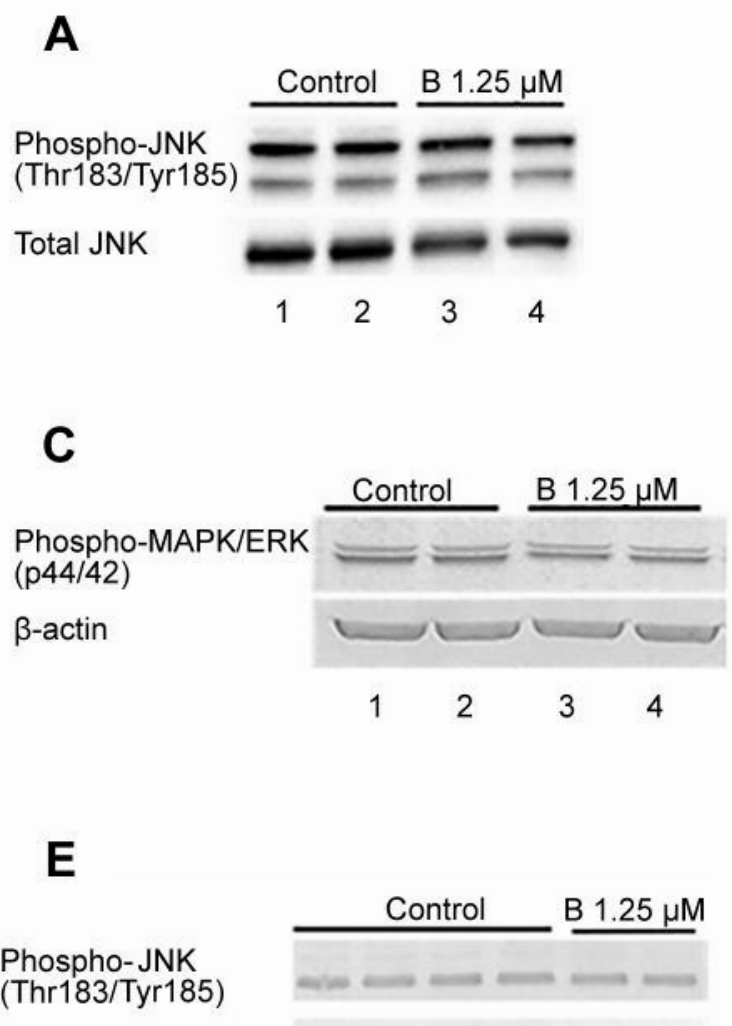

Total JNK

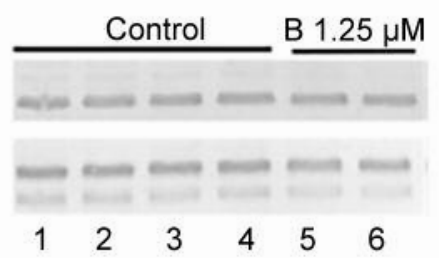

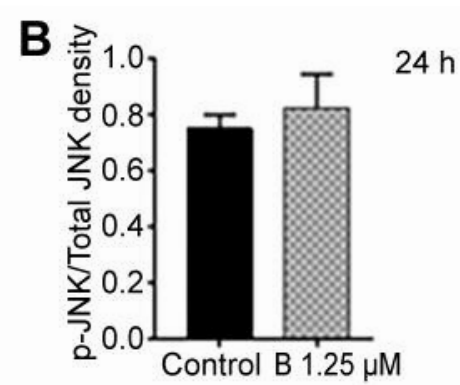

D
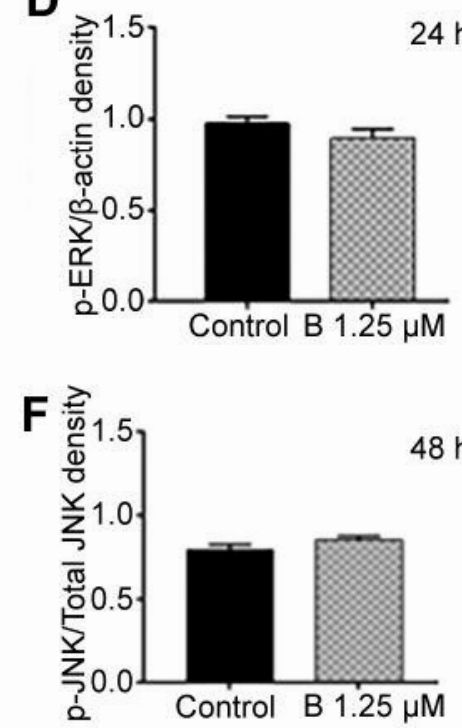

Figure 6. Effect of compound $\boldsymbol{B}$ on JNK or MAPK/ERK phosphorylation. (A) WBrasl cells were treated with vehicle or $1.25 \mu M$ compound $\boldsymbol{B}$ for $24 \mathrm{~h}(\mathrm{~h})$ following treatment and western blot analysis was conducted on whole-cell lysates. Antibodies are specific for phospho-JNK (thr183/tyr185)/ Total JNK (B) Results represent at least two independent experiments for each treatment. (C and D) Effect of compound B on MAPK/ERK in H2009 cells at $24 \mathrm{~h}$ following treatment. C) Immunoblotting of whole lysates shows the effect of compound $\boldsymbol{B}$ on ERK (p44/42) phosphorylation (top lanes) or $\beta$-actin (bottom lanes). Treatment groups were: vehicle (lanes 1-2), $1.25 \mu M$ compound $\boldsymbol{B}$ (lanes 3-4). (E and F) Effect of compound $\boldsymbol{B}$ on JNK in $H 2009$ cells at 48 h following treatment. E) Western blots show the effect of compound $\boldsymbol{B}$ on JNK (Thr183/Tyr185) phosphorylation (top lanes) or total JNK (bottom lanes). Treatment groups were: vehicle (lanes 1-4), $1.25 \mu \mathrm{M}$ compound B (lanes 5-6). Densitometric quantifications of bands shown in the graphs to the right $(B, D, F)$ represent the mean $\pm S . D$. $(p>0.05$ compared to control).

demonstrate that compound $\mathbf{B}$ down-regulates the Akt signaling pathway downstream of Akt in ras-transformed WBras1 cells.

In H2009 cells at $24 \mathrm{~h}$ (Figures 5A and B), compound B decreased phosphorylation of the downstream effector, P70S6 kinase, at $1.25 \mu \mathrm{M}$ and $1.5 \mu \mathrm{M}$ by approximately 80 and $85 \%$, respectively. In under $24 \mathrm{~h}$, compound $\mathbf{B}$ decreased phosphorylation of MDM2, GSK3 $\beta$ and P70S6K in H2009 cells at $2.5 \mu \mathrm{M}$ (Figures $5 \mathrm{C}-\mathrm{H}$ ). MDM2 phosphorylation showed no response at the 1-h time point but was decreased at $4 \mathrm{~h}$ (Figures $5 \mathrm{C}$ and D). These results confirm that the Akt pathway can be down-regulated by compound $\mathbf{B}$ as early as 1-h treatment time and that the onset of the effect of compound $\mathbf{B}$ on phosphorylation of Akt downstream effectors in H2009 cells was different for one of the effectors.

Effect of compound $\boldsymbol{B}$ on other signaling pathways. Figures $6 \mathrm{~A}$ and $\mathrm{B}$ show that there was no significant effect of compound B on phosphorylation of JNK at Thr183/185 in WBras 1 cells at $24 \mathrm{~h}$ at concentrations that were effective in reducing phosphorylation of Akt and downstream effectors. This lack of effect was also seen in H2009 carcinoma cells (Figures 6E and F). Figures 6C and D show that compound B also had no significant effect on MAPK/ERK phosphorylation at the Thr37/46 activation site in H2009 cells. These results suggest that compound B shows specificity for the Akt pathway. 


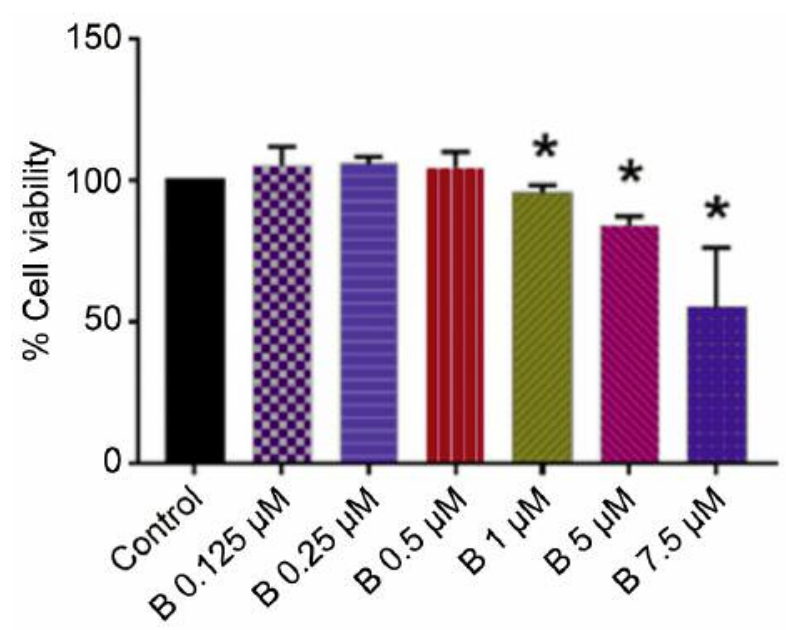

Figure 7. Effect of compound $\boldsymbol{B}$ on cell viability in human lung cancer cells. Cells were treated with $0.125 \mu M, 0.5 \mu M, 1 \mu M, 5 \mu M$, or $7.5 \mu M$ of compound $\boldsymbol{B}$ for 22 h. Percentage of survival of $H 2009$ cells was measured using the MTT assay. Data are expressed relative to the mean optic density $(360 \mathrm{~nm})$ of vehicle-treated cells, which was defined as 100\%. The graph shown represents the mean $\pm S D(n=4),(p<0.05$ compared to control).

Effect of compound $\boldsymbol{B}$ on cell viability in human lung carcinoma cells. Results from an MTT cell viability assay in Figure 7 show that compound $\mathbf{B}$ reduces cell viability in human lung carcinoma cells (H2009) in a dose-dependent manner. In this cell line, the cell viability decreased to approximately $95 \%$, $85 \%$, and $55 \%$ compared to control level after 1,5 and $7.5 \mu \mathrm{M}$ of compound $\mathbf{B}$ treatment for $22 \mathrm{~h}$, respectively.

Compound $\boldsymbol{B}$ is not a direct inhibitor of Akt kinase. To determine whether $\mathbf{B}$ directly inhibits Akt kinase at the active site, we monitored its activity using a cell-free based assay. Figure 8 indicates that compound $\mathbf{B}$ has no direct inhibitory effect on Akt activity compared to the positive control active site inhibitor, staurosporine. These results suggest that compound $\mathbf{B}$ modulates Akt activity by binding to a site different from the active site on Akt or an upstream target to cause decreased phosphorylation of Ser473 and Thr308.

Computational chemistry. As previously outlined, compounds $\mathbf{B}, \mathbf{C}$, and $\mathbf{D}$ were identified by pharmacophore molecular modeling using the Schrödinger software (21). Without knowing the bioactive conformation or the small molecule/macromolecular target interactions, the extended all anti form for the non-ionized structures A-D were calculated using different basis sets at various levels of theory. Figure 9 shows the extended forms of $\mathbf{A}$ and $\mathbf{B}$. The structures were geometry optimized using the Møller-Plesset (MP2) perturbation theory with the 6-31G(d) basis set using Spartan 16 (22). Frequency calculations were carried out to ensure that minimum energy structures were identified.

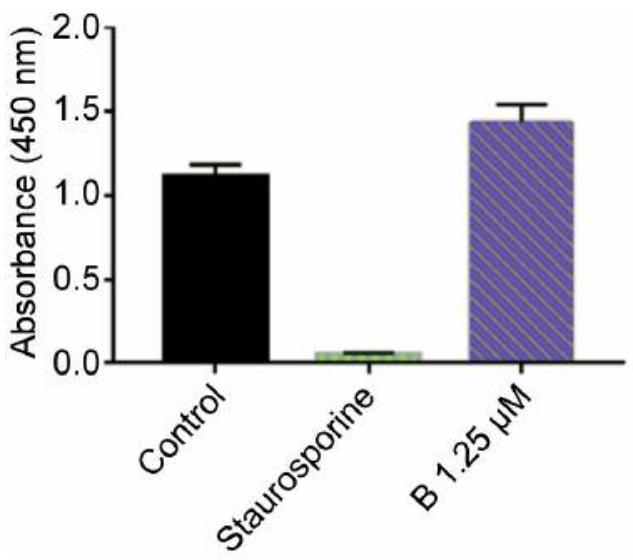

Figure 8. Effect of compound $\boldsymbol{B}$ on Akt kinase activity in a cell-free based assay. The assay was performed as per the assay instructions. Wells were treated with vehicle, $10 \mu \mathrm{M}$ staurosporine, or $1.25 \mu \mathrm{M}$ compound B. The level of inhibition was determined by comparing the vehicle (DMSO) or positive inhibitory control, staurosporine, to compound $\boldsymbol{B}$. Data are expressed relative to the mean optic density $(450 \mathrm{~nm})$. The graph shown represents the mean $\pm S D(n=4),(p<0.05)$.
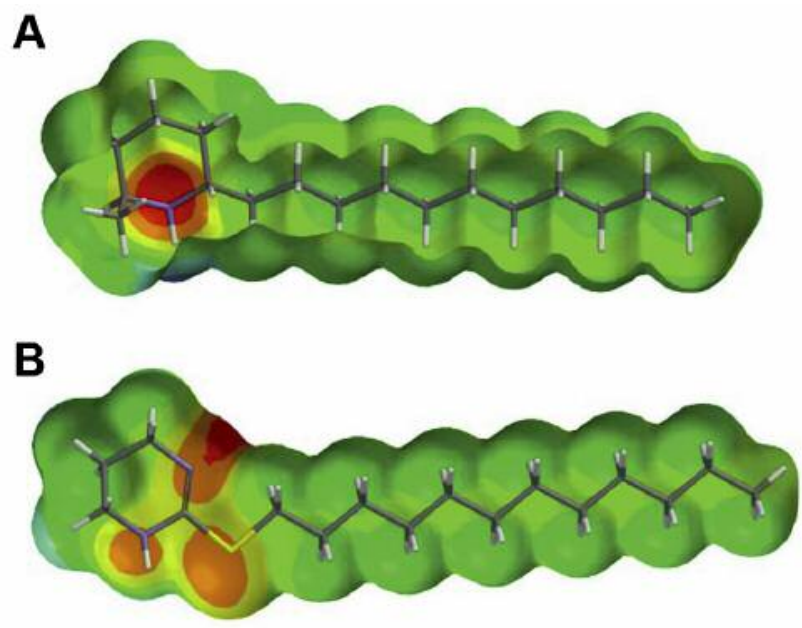

Figure 9. Compounds $\boldsymbol{A}$ and $\boldsymbol{B}$ in their non-ionized form with their side chains in the all anti conformation are displayed with an electrostatic potential map that was generated using the molecular modeling software Spartan16. The colors represent the range of electronegativity, where red and blue represents the more electronegative and electropositive regions, respectively. The two structures were geometry optimized using the Møller-Plesset (MP2) perturbation theory with the 6-31G(d) basis set in Spartan16. Compared to compound A (solenopsin $A)$, compound $\boldsymbol{B}$ is more polar with larger regions of electronegativity.

The overall surfaces of $\mathbf{A}$ and $\mathbf{B}$ are similar, which suggests similar binding modes to the macromolecular target(s) in the Akt pathway. As expected, based on the structural differences, B is calculated to have a larger polar surface area $\left(18.176 \AA^{2}\right)$ 
compared to $\mathbf{A}\left(12.015 \AA^{2}\right)$. The $\log P$ values are calculated in Spartan16 to be 5.48 and 5.80 for $\mathbf{A}$ and $\mathbf{B}$, respectively. Our working assumption is that the greater electrostatic charge distribution present in $\mathbf{B}$ in its ionized form enhances its interactions and inhibitory effects.

\section{Discussion}

The results presented in this study demonstrate that the novel CADD agent, compound $\mathbf{B}$, inhibits the activation of Akt in WBras1 and H2009 cells, as assessed by monitoring the Thr308 phosphorylation site on Akt. We also demonstrate that compound $\mathbf{B}$ decreased phosphorylation of downstream effectors of Akt in both cell types. These include MDM2, 4EBP1, GSK3 $\beta$ and P70S6K. Evidence presented indicates that compound $\mathbf{B}$ induces inhibitory effects on the ability of Akt target proteins to propagate signals, which likely contributes to its anti-proliferative effects in H2009 cells (20).

The investigational compound used in this study is structurally related to solenopsin, which has been characterized as an Akt pathway inhibitor (19). Compound $\mathbf{B}$ was effective in down-regulating the Akt pathway, as measured by the degree of Akt Ser473 (20) and Thr308 phosphorylation, at a >5-fold lower concentration compared to solenopsin. It was reported previously that solenopsin antagonizes Akt, leading to inhibitory effects on angiogenesis (19). It is predicted that compound $\mathbf{B}$ would also inhibit angiogenesis, based on the similar structure and its ability to inhibit the Akt pathway.

In the present study, we first examined the phosphorylation of Akt and total Akt kinase levels in ras-transformed epithelial cells (WBras1) by western immunoblot analysis. Decreased levels of phospho-Akt at Thr308 by compound $\mathbf{B}$ and unaltered levels of total Akt were observed. Similar results were obtained from monitoring compound $\mathbf{B}$ in human lung carcinoma cells. A dose-dependent study of compound B in H2009 cells following a 24-h treatment revealed that concentrations as low as $0.5 \mu \mathrm{M}$ inhibited Akt activation at both Thr308 and Ser473 sites. Examination of downstream pathway inhibition showed significant decreases in phosphorylation levels of MDM2, 4EBP1 and P70S6K in both ras-transformed epithelial and human lung carcinoma cells, with GSK- $3 \beta$ additionally affected in the human carcinoma cells.

The inhibition of MDM2 may be indicative of compound B's ability to disrupt MDM2's interaction with the tumor suppressor, p53 and thus prevent its degradation. This action promotes the normal regulation of cell growth and cell proliferation (23). GSK3 $\beta$ is a substrate of Akt kinase and is inhibited when phosphorylated at the Ser9 site (3). We found that the phosphorylation levels of GSK3 $\beta$ in compound Btreated H2009 cells decreased, suggesting its activation, which mediates the inhibition of glycogen synthesis (3). The reduction in phosphorylation levels observed in 4E-BP1 and P70S76K are indicative of the down-regulation of mTORC1 (24).
We also monitored compound B's ability to affect other signaling pathways. The JNK pathway is known to be activated when the cell undergoes apoptosis or stress (25). Compound $\mathbf{B}$ did not significantly alter phosphorylation of JNK at the Thr183/Y185 activation site at $24 \mathrm{~h}$ in WBras 1 or $\mathrm{H} 2009$ cells. The lack of an effect of the compound $\mathbf{B}$ on JNK is in contrast with reported effects on phosphorylation of this protein by the structurally-related compound, perifosine. Specifically, in a study examining the cytotoxicity effect of perifosine, JNK was evaluated for its role in perifosine-induced cell death and increased cytotoxicity was reported in perifosine-treated cells (17).

We also examined the effect of compound $\mathbf{B}$ on ERK signaling transduction in $\mathrm{H} 2009$ cells. This protein regulates various cytosolic and nuclear proteins, which includes transcription factors of which many are involved in cell proliferation, survival, and metastasis $(26,27)$. At $24 \mathrm{~h}$ compound $\mathbf{B}$ had no effect on the phosphorylation of ERK at the p44/42 activation sites, which highlights its specificity for the Akt pathway.

As evidenced by our results, compound B suppressed activation of Akt and robustly down-regulated the downstream effectors of Akt: i) MDM2, ii) GSK3 $\beta$, iii) P70S6K, and iv) 4EBP-1. The previous assessment of cellular proliferation in compound B-treated-H2009 cells revealed that it induces profound inhibitory effects on the proliferation of tumor cells at sub-micromolar concentrations (20). Further characterization of our investigational compound involved evaluating its ability to directly inhibit Akt kinase using a cell-free based assay. Using the latter, we concluded that compound $\mathbf{B}$ is not a direct Akt inhibitor at the active site.

In summary, our results demonstrate that compound $\mathbf{B}$ inhibits Akt activation and suppresses downstream target proteins along the Akt pathway in ras-transformed rat liver epithelial cells and human lung carcinoma cells. This may occur by allosteric inhibition of Ser473 and Thr308 phosphorylation or by effects on an upstream target, such as mTORC2.

\section{Conflicts of Interest}

None.

\section{Authors' Contributions}

NEU and DFM performed the experiments, NEU and DFM analyzed the data, OFG and JPB performed the pharmacophore modeling, JPB carried out the computational chemistry, DFM, NEU, and JPB wrote the manuscript.

\section{Acknowledgements}

This work was supported by research funds from Mercer University. 


\section{References}

1 Kennedy SG, Wagner AJ, Conzen SD, Jordan J, Bellacosa A, Tsichlis PN and Hay N: The PI 3-kinase/Akt signaling pathway delivers an anti-apoptotic signal. Genes Dev 11: 701-713, 1997. PMID: 9087425. DOI: 10.1101/gad.11.6.701

2 Vivanco I and Sawyers CI: The phosphatidylinositol 3-kinaseAkt pathway in human cancer. Nat Rev Cancer 2: 489-501, 2002. PMID: 12094235 . DOI: $10.1038 / \operatorname{nrc} 839$

3 Manning BD and Cantley LC: AKT/PKB signaling: navigating downstream. Cell 129: 1261-1274, 2007. PMID: 17604717. DOI: 10.1016/j.cell.2007.06.009

4 Shayesteh L, Lu Y, Kuo WL, Baldocchi R, Godfrey T, Collins C, Pinkel, D, Powell B, Mills GB and Gray JW: PIK3CA is implicated as an oncogene in ovarian cancer. Nat Genet 21: 99102, 1999. PMID: 9916799. DOI: 10.1038/5042

5 Sun M, Wang G, Paciga JE, Feldman RI, Yuan Z-Q, Ma X-L, Shelly SA, Jove R, Tsichlis PN, Nicosia SV and Cheng JQ: AKT1/PKBalpha kinase is frequently elevated in human cancers and its constitutive activation is required for oncogenic transformation in NIH3T3 cells. Am J Pathol 159: 431-437, 2001. PMID: 19166270. DOI: 10.1371/journal.pbio.1000017

6 Bellacosa A, Kumar CC, Cristofano AD and Testa JR: Activation of AKT kinases in cancer: implications for therapeutic targeting. Adv Cancer Res 94: 29-86, 2005. PMID: 16095999. DOI: 10.1016/S0065-230X(05)94002-5

7 Nitulescu GM, Margina D, Juzenas P, Peng Q, Olaru OT, Saloustros E, Fenga C, Spandidos DA, Libra M and Tsatsakis AM: Akt inhibitors in cancer treatment: The long journey from drug discovery to clinical use. Int J Oncol 48: 869-885, 2016. PMID: 26698230. DOI: 10.3892/ijo.2015.3306

8 Brown JS and Banerji U: Maximising the potential of AKT inhibitors as anti-cancer treatments. Pharmacol Therapeut 172: 101115, 2017. PMID: 27919797. DOI: 10.1016/j.pharmthera.2016. 12.001

9 Sarbassov DD, Guertin DA, Ali SM and Sabatini DM: Phosphorylation and regulation of Akt/PKB by the rictor-mTOR complex. Science 307: 1098-110, 2005. PMID: 15718470. DOI: 10.1126/science. 1106148

10 Liao Y and Hung M-C: Physiological regulation of Akt activity and stability. Am J Transl Res 2: 19-42, 2010. PMID: 20182580. DOI: $10.3390 /$ cancers 10030078

11 Huang BX and Kim HY: Interdomain conformational changes in Akt activation revealed by chemical cross-linking and tandem mass spectrometry. Mol Cell Proteomics 5: 10451053, 2006. PMID: 16531397. DOI: 10.1074/mcp.M600026MCP200

12 Calleja V, Laguerre M, Parker PJ and Larijani B: Role of a novel $\mathrm{PH}-$ kinase domain interface in $\mathrm{PKB} / \mathrm{Akt}$ regulation: structural mechanism for allosteric inhibition. PLoS Biology 7: e1000017, 2009. PMID: 19166270. DOI: 10.1371/journal.pbio.1000017

13 Alessi DR, Andjelkovic M, Caudwell B, Cron P, Morrice N, Cohen $\mathrm{P}$ and Hemmings BA: Mechanism of activation of protein kinase B by insulin and IGF-1. EMBO J 15: 6541-6551, 1996. PMID: 8978681 .

14 Alessi DR, James SR, Downes CP, Holmes AB, Gaffney PR, Reese CB and Cohen P: Characterization of a 3-phosphoinositide-dependent protein kinase which phosphorylates and activates protein kinase $\mathrm{B} \alpha$. Curr Biol 7: 261-269, 1997. PMID: 9094314.
15 Mora A, Komander D, van Aalten DMF and Alessi DR: PDK1, the master regulator of AGC kinase signal transduction. Semin Cell Dev Biol 15: 161-170, 2004. PMID: 15209375. DOI: 10.1016/j.semcdb.2003.12.022

16 Haar EV, Lee S, Bandhakavi S, Griffin TJ and Kim DH: Insulin signalling to mTOR mediated by the Akt/PKB substrate PRAS40. Nat Cell Biol 9: 316-323, 2007. PMID: 17277771. DOI: $10.1038 / \mathrm{ncb} 1547$

17 Hideshima T, Catley L, Yasui H, Ishitsuka K, Raje N, Mitsiades C, Podar K, Munshi, NC, Chauhan D, Richardson P and Anderson KC: Perifosine, an oral bioactive novel alkylphospholipid, inhibits Akt and induces in vitro and in vivo cytotoxicity in human multiple myeloma cells. Blood 107: 4053-4062, 2006. PMID: 16418332. DOI: 10.1182/blood-2005-08-3434

18 Arbiser JL, Bowen JP and Watkins EB: Solenopsin and derivatives, therapeutic compositions; and methods related thereto. U.S. Patent No. 9, 592, 226, 2017.

19 Arbiser JL, Kau T, Konar M, Narra K, Ramchandran R, Summers S, Vlahos C, Ye K, Perry B, Matter W, Fischl A, Cook $\mathrm{J}$, Silver P, Bain J, Cohen P, Whitmire D, Furness S, Govindarajan B and Bowen JP: Solenopsin, the alkaloidal component of the fire ant (Solenopsis invicta), is a naturally occurring inhibitor of phosphatidylinositol-3-kinase signaling and angiogenesis. Blood 109: 560-565, 2007. PMID: 16990598. DOI: 10.1182/blood-2006-06-029934

20 Uko NE, Güner OS, Barnett LM, Matesic DF and Bowen JP: Discovery and biological activity of computer-assisted drug designed Akt pathway inhibitors. Bioorg Med Chem Lett 28: 32473250, 2018. PMID: 30143420. DOI: 10.1016/j.bmcl. 2018.08.006

21 Schrödinger Small-Molecule Drug Discovery Suite 2014-1, Schrödinger, LLC, New York, NY, 2014. Available at: https://www.schrodinger.com/suites/small-molecule-drugdiscovery-suite (Accessed July 17, 2019)

22 Spartan. Irvine CA: Wavefunction, Inc. Available at: https:// www.wavefun.com/products (Accessed July 17, 2019)

23 Kastan MB, Canman CE and Leonard CJ: P53, cell cycle control and apoptosis: implications for cancer. Cancer and Metastasis Rev 14: 3-15, 1995. PMID: 7606818.

24 Watanabe R, Wei L and Huang J: mTOR signaling, function, novel inhibitors, and therapeutic targets. J Nucl Med 52: 497500, 2011. PMID: 21421716. DOI: 10.2967/jnumed.111.089623

25 Song F, Wang Y, Jiang D, Wang T, Zhang Y, Ma H and Kang Y: Cyclic compressive stress regulates apoptosis in rat osteoblasts: involvement of PI3K/Akt and JNK MAPK signaling pathways. PloS One 11: e0165845, 2016. PMID: 27806136. DOI: 10.1371/journal.pone.0165845

26 Chang L and Karin M: Mammalian MAP kinase signalling cascades. Nature 410: 37-40, 2001. PMID: 11242034. DOI: $10.1038 / 35065000$

27 Heist RS and Engelman JA: SnapShot: non-small cell lung cancer. Cancer Cell 21: 448, 2012. PMID: 22439939. DOI: 10.1016/j.ccr.2012.03.007 